\title{
Past, present and future mathematical models for buildings (ii)
}

Article

Accepted Version

Lu, X., Clements-Croome, D. J. and Viljanen, M. (2009) Past, present and future mathematical models for buildings (ii). Intelligent Buildings International Journal, 1 (2). pp. 131-141. ISSN 1750-8975 doi: https://doi.org/10.3763/inbi.2009.0024 Available at https://centaur.reading.ac.uk/12289/

It is advisable to refer to the publisher's version if you intend to cite from the work. See Guidance on citing.

To link to this article DOI: http://dx.doi.org/10.3763/inbi.2009.0024

All outputs in CentAUR are protected by Intellectual Property Rights law, including copyright law. Copyright and IPR is retained by the creators or other copyright holders. Terms and conditions for use of this material are defined in the End User Agreement.

\section{www.reading.ac.uk/centaur}

\section{CentAUR}

Central Archive at the University of Reading

Reading's research outputs online 


\title{
Past, present and future mathematical models for buildings FOCUS ON INTELLIGENT BUILDINGS (PART 2)
}

\author{
Xiaoshu Lu 1,2,", Derek Clements-Croome³, Martti Viljanen² \\ ${ }^{1}$ Centre of Musculoskeletal Disorders, Finnish Institute of Occupational Health, Topeliuksenkatu \\ 41 a A, FIN-00250 Helsinki, Finland \\ ${ }^{2}$ Laboratory of Structural Engineering and Building Physics, Helsinki University of Technology, \\ PO Box 2100, FIN-02015 HUT, Finland \\ ${ }^{3}$ School of Construction Management and Engineering, Whiteknights, Reading University, \\ PO Box 219, Reading RG6 6AW, UK \\ Corresponding author. E-mail:xiaoshu@cc.hut.fi
}

\begin{abstract}
This article is the second part of a review of the historical evolution of mathematical models applied in the development of building technology. The first part described the current state of the art and contrasted various models with regard to the applications to conventional buildings and intelligent buildings. It concluded that mathematical techniques adopted in neural networks, expert systems, fuzzy logic and genetic models, that can be used to address model uncertainty, are well suited for modelling intelligent buildings. Despite the progress, the possible future development of intelligent buildings based on the current trends implies some potential limitations of these models. This paper attempts to uncover the fundamental limitations inherent in these models and provides some insights into future modelling directions, with special focus on the techniques of semiotics and chaos. Finally, by demonstrating an example of an intelligent building system with the mathematical models that have been developed for such a system, this review addresses the influences of mathematical models as a potential aid in developing intelligent buildings and perhaps even more advanced buildings for the future.
\end{abstract}

Keywords: buildings; intelligent buildings; mathematical modelling

\section{STRENGTHS AND LIMITATIONS OF THE APPROACHES}

These models can be classified in several ways, including the one described in the first part of this article, which broadly described as physical and uncertainty models. To better summarize the strengths and limitations of the applied approaches, we classify these models as physical, statistical and knowledge/logic-based models below, based on their mathematical approaches. As have been seen, each type of model has its own characteristics and can be applied in different situations. The physical models are based on the inherent physical principles which help understand physical processes. Very often, even if the model solution is not available from a physical model, the model can still provide important information about the degrees and nature of intransient coupling among different phenomena. A physical model can show inner parts that are normally not visible in statistical and knowledge/logic-based models. Therefore, most successful models for conventional buildings are physical-based models as reviewed in the previous sections. However, with the increased complexity of intelligent building systems, the physical mechanisms for the systems are too complex and not completely understood. An oversimplified physical model may 
lead to non-realistic predictions. Hence, statistical and knowledge/logic-based models are often adopted which may rely strongly on the experiments and be based on the input-output relationship from the experiments.

A typical feature of statistical and knowledge/ logic-based models is to build model equations from the training part of the experiments and then to apply the model to predict the outcome in the testing part of the experiments. Generalization of the input-output relationship can be difficult due to the lack of physical background in the models. Nowadays, a wide variety of commercial software for statistical models is available. The statistical models are easier to establish and understand than the physical and knowledge/logic-based models. They have been used for intuitive or qualitative analysis of buildings. However, existing applications of statistical models are limited to linear models, and the interpretability of models may be low (Kristensen and Hansen, 2004). Complex systems in intelligent buildings exhibit a rich variety of non-linear dynamics where the experiments, if available, have non-linear characteristics. Statistical models are probably unable to capture the non-linearity in such systems. Above all, experiments are imprecise or even unavailable in human behaviour-based systems in intelligent buildings. Imprecision is different from variation. Statistical models are not suitable to describe the imprecision.

Therefore, intelligent and complex systems for intelligent buildings can only be modelled or configured using knowledge/logic-based models in many cases as we have summarized in previous sections that knowledge-based uncertainty models are a plausible approach to modelling intelligent building systems. Broad applications of such models have been demonstrated in the first part of this a ricle.

Despite their wide applications, the possible future development of conventional and intelligent buildings based on the current trends implies some potential limitations of the knowledge/logic-based models. It is recognizable that logic has driven the overall approach of such models. For a long time people have believed that intelligence is equivalent to logic. The foundation of logic was a law of excluded middle: a statement is either true or false, and any middle alternative is excluded (Aristotle and Organon, 1995). The 'law' entailed a contradiction between exactness of the law of excluded middle and vagueness of language. Logic inventor and founder, Aristotle, and Boole corrected the contradiction and a new logic branch was born as formal lo g i c .

Today, it is generally believed that logic is not a fundamental mechanism of thinking, or intelligence, but the result of the mind's operation (Perlovsky, 2006). Therefore, these models, relying on logic, have encountered some difficulties over the years, mainly summarized as 'combinatorial complexity', which is related to the fundamental inconsistency of logic. Such inconsistency can be demonstrated with Russell's paradox and Gödel's proof (Gödel, 1986) as described in the 'Knowledge-based models' section of part one of this paper (see issue 1(1)).

\section{POSSIBLE FUTURE DIRECTIONS OF MATHEMATICAL MODELS}

Having discussed the strengths and limitations of the applied approaches and following the logical progression of this article, we propose future or potential models. The trend in Box 1 in part one of this paper (see issue 1(1)) shows that future mathematical models should have capacities to model higher degrees of intelligence in order to cope with more advanced building systems. We have summarized how previous knowledge-based models or intelligence models, relying on logic, have problems of combinatorial complexity. Because logic can only provide deductions from principles, but cannot indicate what principles should be, logic is not a fundamental mechanism of mind, and not quite equivalent to intelligence.

With the advancement of bio-information processing techniques, the modelling of higher human cognitive abilities, interaction between environmental and conceptual issues, and unification of language and cognitive is becoming possible for example with theories of qualitative behaviour, mathematical linguistics, cybernetics, computational intelligence and semiotics, among others. 
The abstract level of these models includes approaches of synergetics, chaos and cellular automata, for example (Petri, 1996). Among these potential models and mathematical approaches we are particularly interested in discussing semiotics and chaos. Discussing all of these future models and approaches is beyond the scope of this article. The following subsections will give insights into applications of semiotics and chaos in the intelligent buildings field.

\section{SEMIOTICS}

\section{Semiotics in general}

Semiotics is the study of signs (symbols) (Morris, 1955). There are three levels of semiotics concerned with signs and relations (to other signs, to objects, etc) (Cherry, 1966). They are elaborated in the following way (Peirce, 1933; Morris, 1955; Eco, 1976, 1984):

1. Syntactics deals with signs and their relations to other signs.

2. Semantics deals with the relation of signs to objects, or the interpretation of signs as their meanings.

3. Pragmatics deals with interferential meaning including subtler aspects of communication expressed through indirection and through social contexts.

These three levels have been called representation, object and interpretation by Peirce (1933). They can be characterized to define corresponding levels in different application areas. We shall present the control- semiotic perspective using a conceptualized control system model of intelligent buildings. Our approach is inspired somewhat by Joslyn (2001) and de Souza (2005).

Applications of semiotics in intelligent building research

We shall show how semiotics can contribute to bring about an unusual, intellectual and new approach to intelligent buildings. For illustration purposes, the control system model of intelligent buildings given in the part 1 of this article (see issue 1(1)) is conceptualized as a demonstration example (Figure 1). We seek to study the controls from a systems-cybernetics-semiotics perspective relying on information concepts.

Figure 1 can be further conceptualized as a classically structural view shown in Figure 2

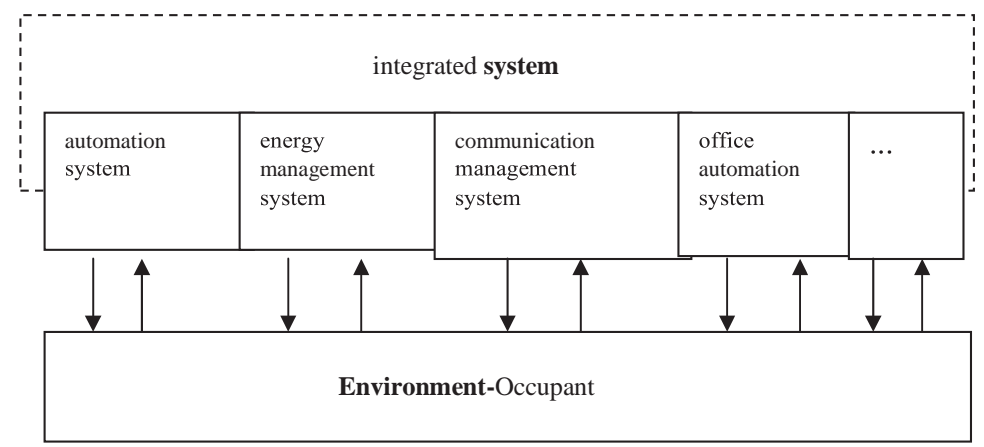

FIGURE I Conceptualized control system model of intelligent buildings 


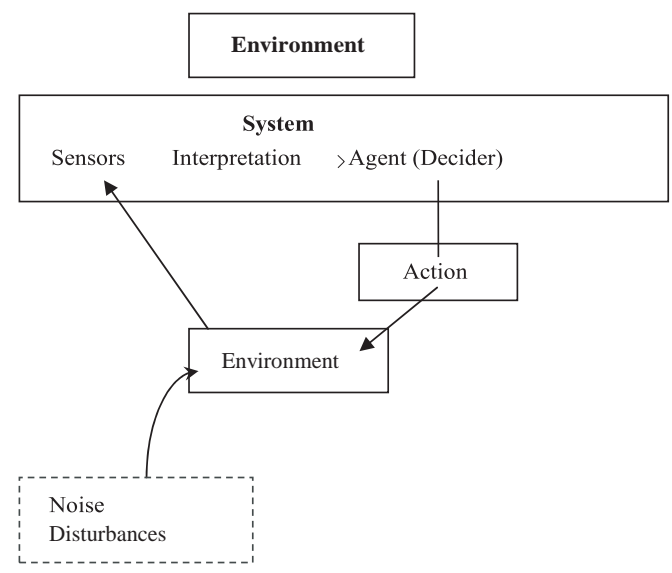

FIGURE 2 Structural view of the control system presented in Figure 1

(Joslyn, 2001). The sensors interact with the environment to produce an interpretation, which is passed to the agent, which makes a decision to choose an action for the environment. It should be stressed that in good control, the system must form a negative feedback loop, as in Figure 2.

We now sketch our understanding of semiotics in relation to the interplay between the system and the environment-occupant in the control system. First, we take sensor measures to be signs. According to the definition of semiotics given by Eco (1976), the following relations between semiotics and the system structure can be found: signs and sensor measures; unlimited semiosis and agent decider; signs and action. The system gives an action based on the 'interpretation' of the relation between environment (sensor measures) and agent. Going deeper into the theory, we find that in the context of semiotics, a sign can be anything to mean something. This 'something' does not necessarily have to exist (Eco, 1976) and may be abstract. In fact, the 'interpretation' process is itself a sign with ongoing 'interpretation' for the action (a kind of unlimited semiosis) (de Souza, 2005). In other words, in semiotics the sign or 'measure' is not a static and definitive entity; hence, the interpretative process is an evolving process. As a consequence, we expect that the dynamic relationship, governed by 'rule' and 'law', among environment, agent and action can be determined and built up with semiotics models.

For this purpose, in Figures 1 and 2, the candidates for the environment-occupant (of intelligent building) have to be identified first, which, for example, may take different levels ranging from individual, organization to society levels. Semiotics analysis can reveal patterns of environment-occupants' meanings, or ontological dependency, to support their semiosis. The pattern means ontological classes of candidates. Second, the learning-adaptation process has dynamic features under different ongoing states determined by various environmental or other factors, such as policy changes due to various contingent reasons. The snapshots of the learning- adaptation process are not necessarily consistent with each other, and semiotics analysis can reveal dynamic features of the process. The dynamic features mean 'rules' and 'laws' which, for example, can characterize dynamically invariant behaviours of the candidates. Such 'dynamically invariant behaviours' may be structurally represented as a chart, called an ontology chart.

Noy et al. (2007) presented approaches to help understand the interplay between people and intelligent buildings using semiotics, aiming at increasing human satisfaction and also lowering energy consumption. The environmental candidates included person, organization and society, and the building system consisted of building facilities and actuator sensors. 


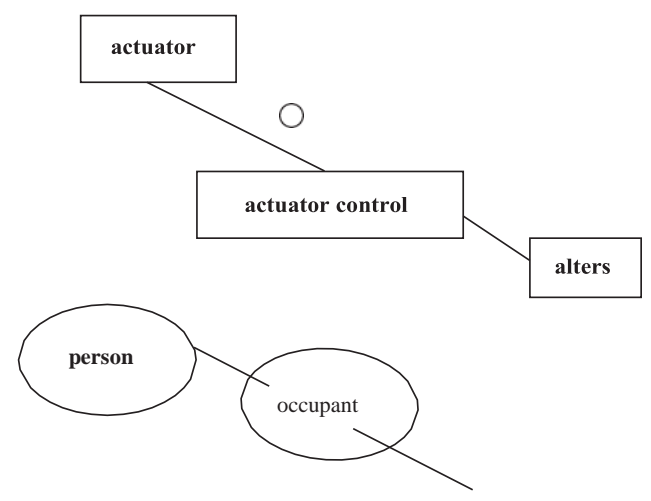

FIGURE 3 Illustration of part of an ontology chart (Noy et al., 2007)

The authors first illustrated how building design can communicate with users at overall and individual levels, and addressed ambiguity problems in communications. They then proposed organization semiotics, a branch of semiotics which has specific concepts and methods for examining communications and organizations, for building/people analysis. Such techniques provide a basis for personalizing intelligent buildings. The final ontology chart was generated for a semantic temporal database, see Figure 3 .

More potential applications of semiotics in intelligent building research

Semiotics has been applied widely. As westressed previously, we can freely choose anything in the system as signs and the 'interpretation' process is continuous. For example, in the control system in Figure 2, typically much more can be done with the sensor measures than simply passing them to the agent for decision-making. It is possible to augment a computer control system between the sensors and agent as a new relation. The computer control system can perform any role, such as cognition, information processing or knowledge development. Semiotics models can then be applied to analyse this new relation. In principle, semiotics analysis can be applied to any of the relations in the system, say, computation or mathematical modelling of the system components. However, these functional relations are normally studied using conventional techniques. The non-functional ones are difficult to specify and therefore semiotics technique is employed for modelling purposes (Clements- Croome, 2004).

It is worth mentioning that due to the wide application of semiotics, there is a very rich set of terminology among the literature papers. Based on Liu (2000), semiotics analysis procedure can be generalized as four-phase analysis. 'Semantic analysis' and 'norm analysis' indicate the two- step procedures described in our context. 'Norms' are equivalent to 'rules' and 'laws' here. The concept of 'affordance' is an effective way of describing the potential functions (what the system can afford) and the relationship between the functions (as some functions ontologically depend on others), which is associated with the action in our control system.

Compared with the 'intelligent buildings' described in previous subsections, the crucial difference about the intelligent buildings in this subsection is that problems can be solved by learning-adaptation abilities springing from an epistemology of practice from the environment- occupant that can help the systems to deal with the inherent complexity of situations where there are more variables - kinds of possible moves, rules, laws and interrelationships - than can be represented in a finite model. It is easy to see that such intelligent buildings have a much higher degree of intelligence than those discussed previously. From a modelling point of view, the semiotics model is an infinite model which imitates human cognition. The philosophical hypothesis behind the semantic model is that 
correct information, 'rules' or 'laws', can be reached via meaning and interpretation through a recursive process.

Again we would like to emphasize that this subsection demonstrates how semiotics can contribute to bringing about unusual and more intellectual approaches to intelligent buildings. The conclusions we reached are abstracted and general, and can be applied to a wide variety of intelligent building studies. Semiotics has been applied in many fields such as language, literature, film and theatre, as well as building design. However, a critical literature review shows that the only semiotics directly applied in the intelligent building field recently seems to be organizational semiotics, which is a branch of semiotics (Clements- Croome, 2004). Therefore, we chose in this section to demonstrate the effectiveness and potential value of semiotic methods in complement to the approaches discussed in the previous section. It is not difficult to see that our demonstration involved more than just methodology reviews.

\section{CHAOS THEORY}

Given the need for a new understanding of modelling higher human cognitive abilities and non-linear dynamic systems, we need to introduce chaos theory. An overview of chaos theory is given by Gleick (1988).

\section{Chaos theory in general}

While there is no exact definition for chaos, chaos theory is characterized by the so-called 'butterfly effect': that small changes in initial conditions of dynamic events can have radically large effects on final outcomes. The sensitivity to initial conditions is measured by a quantity called the Lyapunov exponent. A very interesting feature of chaos theory is that such an unstable phenomenon is not totally out of control, it is non-stochastic.

\section{Fractals}

A chaotic dynamic system has the property that a sequence of $m$ values will generally lie in a restricted area of $\mathrm{m}$ space, rather than at random. This raises the question of 'dimension' of the chaotic system, which has been linked to that of fractal behaviour. Such dimension is defined as a non-integer value.

The basic concept of fractals is that they contain a large degree of self-similarity. They have little copies of themselves buried deep within the original. And they also have infinite detail. For example, the logistic model in population biology (May, 1976) has chaotic behaviour and self-similarity of certain patterns which are fractals.

\section{Strange attractors}

Strange attractors describe the evolution of a dynamic system through time. If, for whatever reason, the starting value, the evolution series or orbits lead to a point, then we call the point an attractor. The evolution series can lie in a restricted space with a certain pattern or geometrical form, like fractals, which are called a strange attractor. For example, the famous Lorenz attractor resembles butterfly wings. Strange attractors characterize the dynamic behaviour of non-linear models into certain order. To investigate the structure of a dynamic system, a slice, known as a Poincarè section, can be taken through strange attractors and reveals a two-dimensional view of the path of the attractor as it passes through the same plane in phase space (Gleick, 1988).

\section{Identification of chaos}

Several tests can be employed to examine the chaotic phenomena of a dynamic system. These tests include fast Fourier transforms, the maximum entropy method, the Lyapunov exponent and the capacity dimension and correction dimension, to cite a few (Chatfield, 1996; Zohrabian et al., 2003; Cerny, 2004). The sensitivity to initial condition is measured by the Lyapunov exponent, which is a measure of the rate at which nearby trajectories in phase space diverge. Chaotic orbits have at least 
one positive Lyapunov exponent and periodic orbits have only one negative Lyapunov exponent.

Potential applications of chaos theory in intelligent building research

Starting from the system modelling point of view, more control systems are characterized by non- linear dynamic features for intelligent buildings. The previous discussion shows that traditional analysis techniques for non-linear system may lead to erroneous results especially when bifurcations occur. Indeed, ingeneral, bifurcations are the course of an additional distinctively non- linear feature in the dynamics. Among other causes, bifurcations may happen because of the possibility of co-existing stable equilibrium positions; each has its own domain of attractors, suddennon-lineartransitioncaused by non-linear hysteresis phenomena, transitions to chaos or chaotic explosions, transitions are qualitatively big for small parameter changes or increase of the dimensionality of the dynamics (Kerschen et al., 2006). These features differentiate clearly the non-linear from the linear dynamics which need chaos theory to identify and analyse the systems.

In addition, the main reason why chaos is of interest is that a chaotic system is sensitive to initial conditions, and a chaotic deterministic system can appear to behave as if it were 'random'. In control of intelligent buildings, it would be of great importance to building researchers if uncertainty or imprecision signals, previously thought to be random, turned out to have deterministic explanations. Furthermore, control application of the 'initial sensitivity' of a chaotic system can be made by a 'tiny adjustment' of the system parameters, which gives possibilities of controlling systems without interfering with their inherent properties. It opens up new markets for control theory. Fradkov and Evans (2005) reviewed methods and application of chaos theory in control engineering and concluded that there are many interesting, yet unsolved, problems of chaos theory for engineering applications. In fact, the topic of chaos has received much attention in many engineering areas but not in the building field. Such a situation is likely to attract the interest of building researchers.

From the modelling point of view, chaos theory has been applied to many fields, such as sociology, economics, cognitive and linguistics science and brain dynamics (Howe and Rabinowitz, 1994; Ruhland and van Geert, 1998; Ikegami and Morimoto, 2003). Modelling and controlling complex systems in intelligent buildings will require new methods to model intelligent systems. Research in these fields will almost certainly result in better understanding of complex phenomena to develop 'more intelligent' intelligent designs.

\section{EXAMPLE}

Hagras and his co-workers (2003) developed and implemented intelligent building agents based on a double-hierarchical fuzzy genetic multi- embedded-agent architecture for an intelligent building. The system is able to learn and adapt to individuals within a building. In the following, we briefly describe the system in a hierarchical way. For details, refer to Hagras et al. (2003).

\section{INTELLIGENCE ARCHITECTURE}

The architecture is a room based system. Each room contains an embedded agent which is responsible for the local control of that room through sensors and effectors. All embedded agents, sensors and effectors are connected together using a network. The distributed artificial intelligence architecture is displayed in Figure 4.

\section{EMBEDDED AGENTS}

The embedded agents are behaviour-based systems. To account for the complex system resulting from a large integrated building, such as the large input, output and many imprecise and unpredictable factors 
including the occupant, the control space is broken down into multiple behaviours and then integrated recommendations are made from the multiple behaviours. Single behaviour and the overall integrated behaviours are implemented with fuzzy process and agents. Consequently, a double-hierarchical fuzzy system is developed and its architecture is shown in Figure 5.

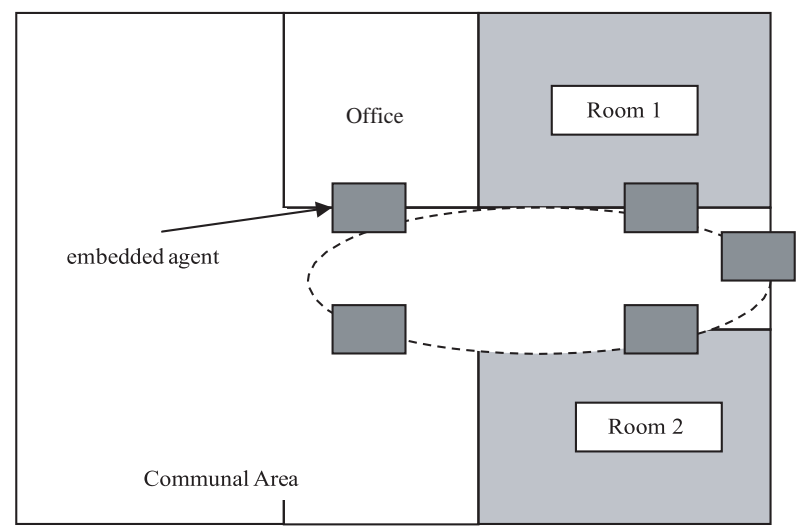

FIGURE 4 The room-based architecture (modified from Hagras et al., 2003)

Now we turn to the behaviour decomposition. It contains the following meta-functions: a safe behaviour, an emergency behaviour and an economy behaviour which are fixed but settable, as well as a set of comfortable behaviours which the system learns from the occupant. The learning is an interactive process: the controller takes actions and monitors these actions to see if they satisfy the occupant or not, until a degree of satisfaction is achieved.

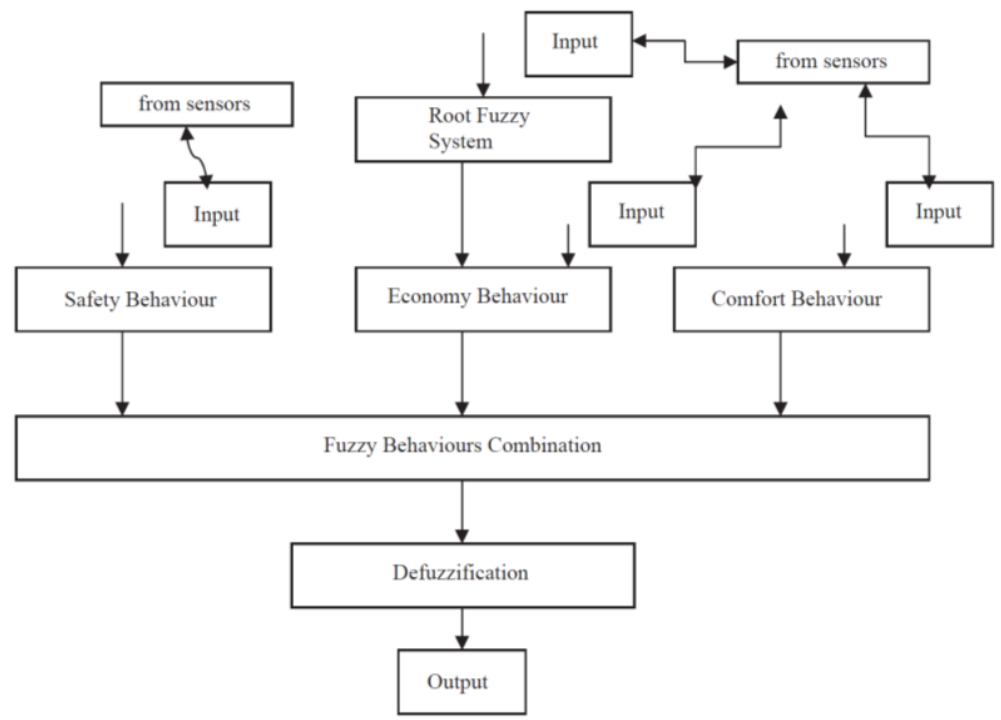

FIGURE 5 The hierarchical fuzzy control system for embedded agents (modified from Hagras et al., 2003) 


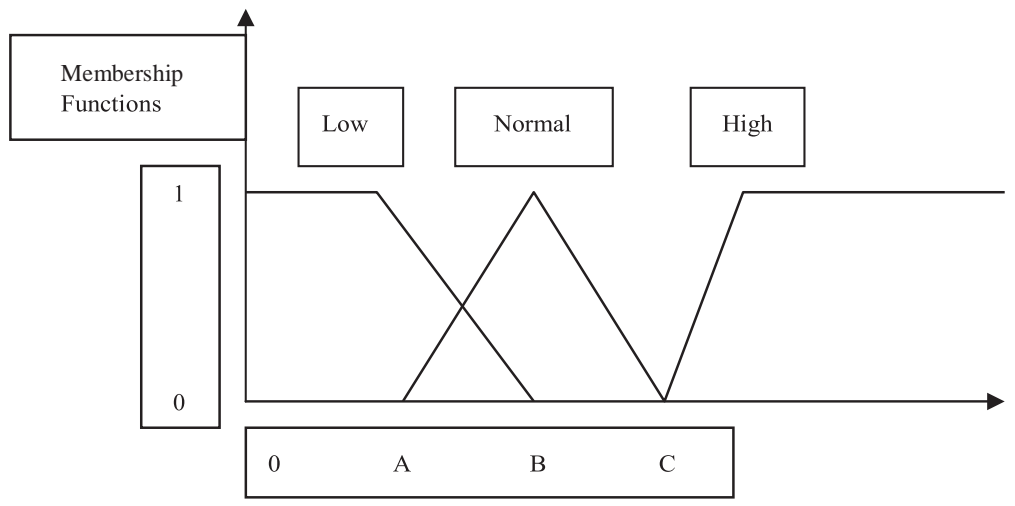

FIGURE 6 An illustration of input membership functions (modified from Hagras et al., 2003)

The behaviours, inside the agent, take their input from sensors and adjust device outputs according to predetermined levels. Each agent has six inputs made up of four environmental variables, each of which has the fuzzy membership function shown in Figure 6, which represents three fuzzy sets.

The system has two outputs whose fuzzy membership functions are presented by seven fuzzy sets (not shown here). The fuzzy membership of integrated behaviours is illustrated in Figure 7 . Mappings from input to output are presented as 'averaged' formulae for simplicity. Each behaviour is treated as an independent fuzzy controller, and then fuzzy behaviour combination is used to obtain a collective fuzzy output which is then defuzzified to obtain a final crisp output. The final output is a mixture of the different behaviour outputs, each weighted by the degree of its importance.

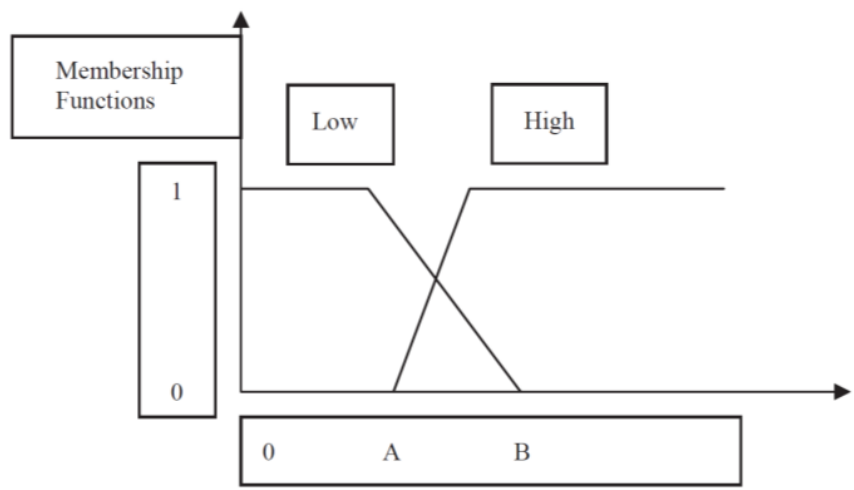

FIGURE 7 An illustration of coordinate parameters' membership functions (modified from Hagras et al., 2003)

\section{GENETICALGORITHM FOR LEARNING AND ADAPTATION TO OCCUPANTS}

A genetic algorithm-based approach is used for learning and adapting the dynamic comfort rule base according to the occupant behaviours. This mechanism operates directly on the fuzzy controller rulesets.

The learning engine within the control architecture is termed an 'associative experience engine' from British patent 99-10539.7. The learning depends on the learning focus which is supplied by the coordinator (the fuzzy engine which weights contributions to the outputs). Interactive learning of the occupant's preferred behaviours is processed through the experience bank by taking control and 
fires the most suitable rule base. It can cope with different occupants' preferred behaviours, such as new occupants and even those with odd behaviours. More techniques concerning the learning engine, such as how to identifying poorly performing rules, fitness determination and credit assignment, memory application and using genetic algorithms to produce new solutions, are also discussed in detail by Hagras et al. (2003). A figure showing the overall embedded agent architecture can also be found in Hagras et al. (2003).

\section{CONCLUSIONS}

This two-part article attempts to find how mathematical models have helped building technology development. By reviewing in parallel the trend of technical development for buildings and mathematical model development, both building systems and mathematical models were studied throughout the whole development process, from conventional to intelligent buildings as a focus, and from first-principle physical models, knowledge-based uncertainty models to future models. The abstract level of the mathematical models were detailed and classified. In order to illustrate the link between the findings of the literature review and practice issues, an example of intelligent building system and the applied models was demonstrated. The major findings are summarized as follows:

- Physical models (or semi-physical models) have played an important role in understanding mechanisms of buildings and generating and testing hypotheses. They are widely applied in conventional building controls.

- Knowledge-based uncertainty models are a plausible approach to modelling intelligent building systems which have ill-definition, uncertainty and multiple objectives characteristics, like human decision-making processes.

- However, the intelligent degree of the knowledge-based uncertainty models should be questioned, as pointed out in this article, in the sense that these models, relying on logic, are related to the fundamental inconsistency of logic, which is not totally equivalent to intelligence.

- Therefore, future work including extending the knowledge-based uncertainty models to those of higher human cognitive abilities, interaction between environmental and conceptual, and unification of language and cognitive is proposed. The abstract level of these models includes approaches of synergetics, chaos and cellular automata. The representative model and approach, semiotics and chaos, were discussed and their potential applications in intelligent buildings were addressed in this article.

- The chief value of semiotics models is the combination of freedom and determinism of the modelling process, which is not possible with any purely physical models. From a modelling point of view, semiotics models are a kind of infinite model which imitates human cognition. Despite the fact that semiotics has been confined to analysis of organizational issues for intelligent buildings in literature, the demonstration here is good enough to justify the usefulness of semiotics as a new theory in the building field which is very promising for models applied in intelligent buildings.

- With regard to the abstract level of higher intelligent models like modelling human cognitive abilities, as well as its direct application to intelligent building research, chaos theory will be quite useful.

Finally, it is concluded from this review that mathematical models have speeded up the design, analysis, construction and operation of buildings and succeeded in supporting new technologies in the construction industry, leading to more technologically advanced intelligent buildings. 


\section{REFERENCES}

Aristotle, B.C.E. and Organon, I.V., 1995, in J. Barnes (ed) Complete Works of Aristotle, Princeton, NJ, Princeton University Press, 18a2819b4, 1011b24-1012a28.

Cerny, A., 2004, 'Introduction to fast Fourier transforms in finance',

The Journal of Derivatives, fall, 73-88.

Chatfield, C., 1996, The Analysis of Time Series, 5th edn., New York, NY, Chapman \& Hall.

Cherry, I.C., 1966, On Human Communication, 2nd edn., Cambridge, MA, MIT Press.

Clements-Croome, D.J. (ed), 2004, Intelligent Buildings: Design, Management and Operation, Thomas Telford.

de Souza, C.S., 2005, 'Semiotic engineering: bring designers and users together at interaction time', Interacting with Computers 17, 317-341.

Eco, U., 1976, A Theory of Semiotics. Bloomington, IN, University Press.

Eco,U., 1984,Semioticsand thePhilosophyofLanguage,Bloomington, IN, Indiana University Press.

Fradkov, A.L. and Evans, R.J., 2005, 'Control of chaos: methods and applications in engineering', Annual Reviews in Control 29, 33-56.

Gleick, J., 1988, Chaos: Making a New Science, New York, Cardinal Book, WilliamHeinemann.

Gödel, K., 1986, in S. Feferman et al. (eds), Kurt Gödel Collected Works, I, Oxford, Oxford University Press.

Hagras, H., Callaghan, V., Colley, M. and Clarke, G., 2003, 'A hierarchical fuzzy-genetic multi-agent architecture for intelligent buildings online learning, adaptation and control', Information Science 150(1-2), 33-57.

Howe, M.L. and Rabinowitz, F.M., 1994, 'Dynamic modelling, chaos, and cognitive development', Journal of Experimental Child Psychology 58 , 184-199.

kegami, T. and Morimoto, G., 2003, 'Chaotic itinerancy in coupled dynamical recognizers. Chaos: An Interdisciplinary', Journal of Nonlinear Science 13(3), 1133-1147.

Joslyn, C., 2001, 'The semiotics of control and modelling relations in complex systems', BioSystems 60, 131-148.

Kerschen, G., Worden, K., Vakakis, A.F. and Golinval, J.C., 2006,

'Past, present and future of nonlinear system identification in structural dynamics', Mechanical Systems and Signal Processing 20(3), 505592.

Kristensen, M. and Hansen, T. 2004, 'Statistical analyses of repeated measures in physiological research: a tutorial', Advances in Physiological Education 28, 2-14.

Liu, K., 2000, Semiotics in Information Systems Engineering, Cambridge University Press.

May, R., 1976, 'Simple mathematical models with very complicated dynamics'. Nature 261, 459-467.

Morris, C.W., 1955, Signs, Language and Behaviour, New York, Braziller.

Noy, P., Liu, K. and Clements-Croome, D.J, 2007, 'Using semiotics to understand the interplay between people and buildings'. Proceedings of Clima, Helsinki.

Peirce, C.S., 1933, in C. Hartshorne and P. Weiss (eds), Collected Papers, Cambridge, MA, Harvard University Press.

Perlovsky, L.I., 2006, 'Toward physics of the mind: concepts, emotions, consciousness, and symbols', Physics of Life Reviews 3, 23-55.

Petri, C.A., 1966, Communication with Automata, Technical Report, RADC-TR-65-377, vol. 1, suppl. 1, New York, Griffiss Air Force Base.

Ruhland, R. and van Geert, P., 1998, 'Jumping into syntax: transitions in the development of closed class words', British Journal of Developmental Psychology 16, 65-95.

Zohrabian, A., Traxler, G., Caudill, G. and Smale, M., 2003, 'Valuing pre-commercial genetic resources: a maximum entropy approach', American Journal of Agricultural Economics 2, 429-436. 